\title{
$m$-Polar Fuzzy Sets: An Extension of Bipolar Fuzzy Sets
}

\author{
Juanjuan Chen, ${ }^{1,2}$ Shenggang $\mathrm{Li}^{1}{ }^{1}$ Shengquan $\mathrm{Ma},{ }^{1}$ and Xueping Wang ${ }^{3}$ \\ ${ }^{1}$ College of Mathematics and Information Science, Shaanxi Normal University, Xian 710062, China \\ ${ }^{2}$ School of Sciences, Xian University of Technology, Xian 710056, China \\ ${ }^{3}$ College of Mathematics and Software Science, Sichuan Normal University, Chengdu 610066, China
}

Correspondence should be addressed to Shenggang Li; shengganglinew@126.com

Received 11 April 2014; Accepted 23 May 2014; Published 12 June 2014

Academic Editor: Jianming Zhan

Copyright (C) 2014 Juanjuan Chen et al. This is an open access article distributed under the Creative Commons Attribution License, which permits unrestricted use, distribution, and reproduction in any medium, provided the original work is properly cited.

\begin{abstract}
Recently, bipolar fuzzy sets have been studied and applied a bit enthusiastically and a bit increasingly. In this paper we prove that bipolar fuzzy sets and $[0,1]^{2}$-sets (which have been deeply studied) are actually cryptomorphic mathematical notions. Since researches or modelings on real world problems often involve multi-agent, multi-attribute, multi-object, multi-index, multi-polar information, uncertainty, or/and limit process, we put forward (or highlight) the notion of $m$-polar fuzzy set (actually, $[0,1]^{m}$-set which can be seen as a generalization of bipolar fuzzy set, where $m$ is an arbitrary ordinal number) and illustrate how many concepts have been defined based on bipolar fuzzy sets and many results which are related to these concepts can be generalized to the case of $m$-polar fuzzy sets. We also give examples to show how to apply $m$-polar fuzzy sets in real world problems.
\end{abstract}

\section{Introduction and Preliminaries}

Set theory and logic systems are strongly coupled in the development of modern logic. Classical logic corresponds to the crisp set theory, and fuzzy logic is associated with fuzzy set theory which was proposed by Zadeh in his pioneer work [1].

Definition 1. An $L$-subset (or an $L$-set) on the set $X$ is a synonym of a mapping $A: X \rightarrow L$, where $L$ is a lattice (cf. [2]). When $L=[0,1]$ (the ordinary closed unit interval with the ordinary order relation), an $L$-set on $X$ will be called a fuzzy set on $X$ (cf. [1]).

The theory of fuzzy sets has become a vigorous area of research in different disciplines including medical and life sciences, management sciences, social sciences, engineering, statistics, graph theory, artificial intelligence, pattern recognition, robotics, computer networks, decision making, and automata theory.

An extension of fuzzy set, called bipolar fuzzy set, was given by Zhang [3] in 1994.

Definition 2 (see Zhang [3]). A bipolar fuzzy set is a pair $\left(\mu^{+}, \mu^{-}\right)$, where $\mu^{+}: X \rightarrow[0,1]$ and $\mu^{-}: X \rightarrow[-1,0]$ are any mappings. The set of all bipolar fuzzy sets on $X$ is denoted by $\mathrm{BF}(X)$.

Bipolar fuzzy sets are an extension of fuzzy sets whose membership degree range is $[-1,1]$. In a bipolar fuzzy set, the membership degree 0 of an element means that the element is irrelevant to the corresponding property, the membership degree $(0,1]$ of an element indicates that the element somewhat satisfies the property, and the membership degree $[-1,0)$ of an element indicates that the element somewhat satisfies the implicit counter-property. The idea which lies behind such description is connected with the existence of "bipolar information" (e.g., positive information and negative information) about the given set. Positive information represents what is granted to be possible, while negative information represents what is considered to be impossible. Actually, a wide variety of human decision making is based on double-sided or bipolar judgmental thinking on a positive side and a negative side. For instance, cooperation and competition, friendship and hostility, common interests and conflict of interests, effect and side effect, likelihood and unlikelihood, feedforward and feedback, and so forth are often the two sides in decision and coordination. In the traditional Chinese medicine (TCM for short), "yin" and 
"yang" are the two sides. Yin is the feminine or negative side of a system and yang is the masculine or positive side of a system. The coexistence, equilibrium, and harmony of the two sides are considered a key for the mental and physical health of a person as well as for the stability and prosperity of a social system. Thus bipolar fuzzy sets indeed have potential impacts on many fields, including artificial intelligence, computer science, information science, cognitive science, decision science, management science, economics, neural science, quantum computing, medical science, and social science (cf. [4-45]). In recent years bipolar fuzzy sets seem to have been studied and applied a bit enthusiastically and a bit increasingly (cf. [4-45]). This is the chief motivation for us to introduce and study $m$-polar fuzzy sets.

The first object of this note is to answer the following question on bipolar fuzzy sets.

Question 1. Is bipolar fuzzy set a very intuitive $L$-set?

The answer to Question 1 is positive. We will prove in this note that there is a natural one-to-one correspondence between $\mathrm{BF}(X)$ and $2(X)$ (for the set of all $[0,1]^{2}$-sets on $X$, see Theorem 5 ) which preserves all involved properties. This makes the notion of bipolar fuzzy set more intuitive. Since properties of $L$-sets have already been studied very deeply and exhaustively, this one-to-one correspondence may be beneficial for both researchers interested in abovementioned papers and related fields (because they can use these properties directly and even cooperate with theoretical fuzzy mathematicians for a possible higher-level research) and theoretical fuzzy mathematicians as well (because cooperation with applied fuzzy mathematicians and practitioners probably makes their research more useful).

We notice that "multipolar information" (not just bipolar information which corresponds to two-valued logic) exists because data for a real world problem are sometimes from $n$ agents $(n \geq 2)$. For example, the exact degree of telecommunication safety of mankind is a point in $[0,1]^{n}(n \approx$ $7 \times 10^{9}$ ) because different person has been monitored different times. There are many other examples: truth degrees of a logic formula which are based on $n$ logic implication operators $(n \geq 2)$, similarity degrees of two logic formulas which are based on $n$ logic implication operators $(n \geq 2)$, ordering results of a magazine, ordering results of a university, and inclusion degrees (resp., accuracy measures, rough measures, approximation qualities, fuzziness measures, and decision performance evaluations) of a rough set. Thus our second object of this note is to answer the following question on extensions of bipolar fuzzy sets.

Question 2. How to generalize bipolar fuzzy sets to multipolar fuzzy sets and how to generalize results on bipolar fuzzy sets to the case of multipolar fuzzy sets?

The idea to answer Question 2 is from the answer to Question 1, intuitiveness of the point-wise order on $[0,1]^{m}$ (see Remark 3), and the proven corresponding results on bipolar fuzzy sets. We put forward the notion of $m$-polar fuzzy set (an extension of bipolar fuzzy set) and point out that many concepts which have been defined based on bipolar fuzzy sets and many results related to these concepts can be
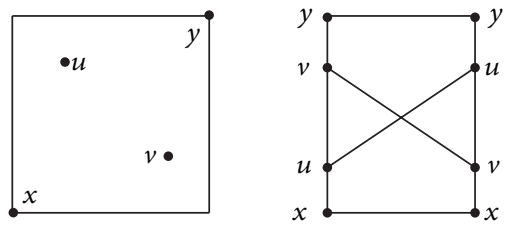

FIGURE 1

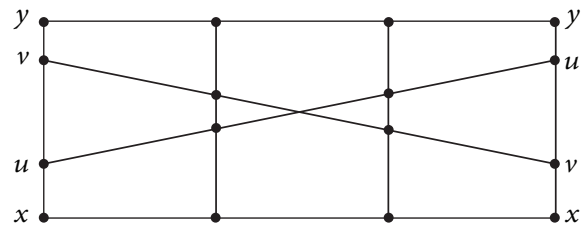

Figure 2

generalized to the case of $m$-polar fuzzy sets (see Remarks 7 and 8 for details).

Apart from the backgrounds (e.g., "multipolar information") of $m$-polar fuzzy sets, the following question on further applications (particularly, further applications in real world problems) of $m$-polar fuzzy sets should also be considered.

Question 3. How to find further possible applications of $m$ polar fuzzy sets in real world problems?

Question 3 can be answered as in the case of bipolar fuzzy sets since researches or modelings on real world problems often involve multiagent, multiattribute, multiobject, multiindex, multipolar information, uncertainty, or/and limits process. We will give examples to demonstrate it (see Examples 9-14).

Remark 3. In this note $[0,1]^{m}(m$-power of $[0,1])$ is considered a poset with the point-wise order $\leq$, where $m$ is an arbitrary ordinal number (we make an appointment that $m=$ $\{n \mid n<m\}$ when $m>0$ ), $\leq$ (which is actually very intuitive as illustrated below) is defined by $x \leq y \Leftrightarrow p_{i}(x) \leq p_{i}(y)$ for each $i \in m\left(x, y \in[0,1]^{m}\right)$, and $p_{i}:[0,1]^{m} \rightarrow[0,1]$ is the $i$ th projection mapping $(i \in m)$.

(1) When $m=2,[0,1]^{2}$ is the ordinary closed unit square in Euclidean plane $R^{2}$. The righter (resp., the upper) a point in this square is, the larger it is. Let $x=\langle 0,0\rangle=$ $\mathbf{0}$ (the smallest element of $\left.[0,1]^{2}\right), u=\langle 0.25,0.75\rangle$, $v=\langle 0.75,0.25\rangle$, and $y=\langle 1,1\rangle$ (the largest element of $[0,1]^{2}$ ). Then $x \leq z \leq y$ for all $z \in[0,1]^{2}$ (especially, $x \leq u \leq y$ and $x \leq v \leq y$ hold). Notice that $u \nless$ $v \not u$ because both $p_{0}(u)=0.25 \leq 0.75=p_{0}(v)$ and $p_{1}(u)=0.75 \geq 0.25=p_{1}(v)$ hold. The order relation $\leq$ on $[0,1]^{2}$ can be illustrated in at least two ways (see Figure 1).

(2) When $m>2$, the order relation $\leq$ on $[0,1]^{m}$ can be illustrated in at least one way (see Figure 2 for the case $m=4$, where $x \leq u \leq y, x \leq v \leq y)$. 


\section{Main Results}

In this section we will prove that a bipolar fuzzy set is just a very specific $L$-set, that is, $[0,1]^{2}$-set. We also put forward (or highlight) the notion of $m$-polar fuzzy set (which is still a special $L$-set, i.e., $[0,1]^{m}$-set, although it is a generalization of bipolar fuzzy set) and point out that many concepts which have been defined based on bipolar fuzzy sets and results related to these concepts can be generalized to the case of $m$ polar fuzzy sets.

Definition 4. An $m$-polar fuzzy set (or a $[0,1]^{m}$-set) on $X$ is exactly a mapping $A: X \rightarrow[0,1]^{m}$. The set of all $m$-polar fuzzy sets on $X$ is denoted by $m(X)$.

The following theorem shows that bipolar fuzzy sets and 2-polar fuzzy sets are cryptomorphic mathematical notions and that we can obtain concisely one from the corresponding one.

Theorem 5. Let $X$ be a set. For each bipolar fuzzy set $\left(\mu^{+}, \mu^{-}\right)$ on $X$, define a 2-polar fuzzy set

$$
\varphi\left(\mu^{+}, \mu^{-}\right)=A_{\mu}: X \longrightarrow[0,1]^{2}
$$

on $X$ by putting

$$
A_{\mu}(x)=\left\langle\mu^{+}(x),-\mu^{-}(x)\right\rangle \quad(\forall x \in X) .
$$

Then we obtain a one-to-one correspondence

$$
\varphi: B F(X) \longrightarrow 2(X)
$$

its inverse mapping $\psi: 2(X) \rightarrow B F(X)$ is given by $\psi(A)=$ $\left(\mu_{A}^{+}, \mu_{A}^{-}\right)(\forall A \in 2(X)), \mu_{A}^{+}(x)=p_{0} \circ A(x)(\forall x \in X)$, and $\mu_{A}^{-}(x)=-p_{1} \circ A(x)(\forall x \in X)$.

Proof. Obviously, both $\varphi$ and $\psi$ are mappings. For each $\left(\mu^{+}, \mu^{-}\right) \in \mathrm{BF}(X)$,

$$
\begin{aligned}
& {[\psi} \\
& {\left[\psi \varphi\left(\mu^{+}, \mu^{-}\right)\right](x)} \\
& \quad=\left\langle p_{0} \circ \varphi\left(\mu^{+}, \mu^{-}\right)(x),-p_{1} \circ \varphi\left(\mu^{+}, \mu^{-}\right)(x)\right\rangle \\
& \quad=\left\langle p_{0}\left(\left\langle\mu^{+}(x), \mu^{-}(x)\right\rangle\right),-p_{1}\left(\left\langle\mu^{+}(x), \mu^{-}(x)\right\rangle\right)\right\rangle \\
& \quad=\left\langle\mu^{+}(x),-\mu^{-}(x)\right\rangle=\left(\mu^{+}, \mu^{-}\right)(x) \quad(\forall x \in X),
\end{aligned}
$$

which means $\left[\psi \circ \varphi\left(\mu^{+}, \mu^{-}\right)\right]=\left(\mu^{+}, \mu^{-}\right)$. Again, for each $A \in$ $2(X)$ and each $x \in X$,

$$
\begin{aligned}
{[\varphi \circ \psi(A)](x) } & =\varphi\left(\mu_{A}^{+}, \mu_{A}^{-}\right)(x) \\
& =\left\langle\mu_{A}^{+}(x),-\mu_{A}^{-}(x)\right\rangle=A(x),
\end{aligned}
$$

which means $\varphi \circ \psi(A)=A$.
Example 6. Let $\left(\mu^{+}, \mu^{-}\right)$be a bipolar fuzzy set, where $X=$ $\{u, v, w, x, y, z\}$ is a six-element set and $\mu^{+}: X \rightarrow[0,1]$ and $\mu^{-}: X \rightarrow[-1,0]$ are defined by

$$
\begin{gathered}
\mu^{+}=\left\{\frac{0.4}{u}, \frac{0.5}{v}, \frac{0.3}{w}, \frac{1}{x}, \frac{1}{y}, \frac{0.6}{z}\right\}, \\
\mu^{-}=\left\{\frac{-0.3}{u}, \frac{-0.6}{v}, \frac{-1}{w}, \frac{-0.2}{x}, \frac{-1}{y}, \frac{-0.5}{z}\right\} .
\end{gathered}
$$

Then the corresponding 2-polar fuzzy set on $X$ is

$$
\begin{gathered}
A_{\mu}=\left\{\frac{\langle 0.4,0.3\rangle}{u}, \frac{\langle 0.5,0.6\rangle}{v}, \frac{\langle 0.3,1\rangle}{w}, \frac{\langle 1,0.2\rangle}{x},\right. \\
\left.\frac{\langle 1,1\rangle}{y}, \frac{\langle 0.6,0.5\rangle}{z}\right\} .
\end{gathered}
$$

In the rest of this note, we investigate the possible applications of $m$-polar fuzzy sets. First we consider the theoretic applications of $m$-polar fuzzy sets. More precisely, we will give some remarks to illustrate how many concepts which have been defined based on bipolar fuzzy sets and results related to these concepts can be generalized to the case of $m$-polar fuzzy sets (see the following Remarks 7 and 8).

Remark 7. The notions of bipolar fuzzy graph (see $[4,45]$ ) and fuzzy graph (see $[46,47]$ ) can be generalized to the convenient (because it allows a computing in computers) and intuitive notion of $m$-polar fuzzy graph. An $m$-polar fuzzy graph with an underlying pair $(V, E)$ (where $E \subseteq V \times V$ is symmetric; i.e., it satisfies $\langle x, y\rangle \in E \Leftrightarrow\langle y, x\rangle \in E$ ) is defined to be a pair $G=(A, B)$, where $A: V \rightarrow$ $[0,1]^{m}$ (i.e., an $m$-polar fuzzy set on $V$ ) and $B: E \rightarrow$ $[0,1]^{m}$ (i.e., an $m$-polar fuzzy set on $E$ ) satisfy $B(\langle x, y\rangle) \leq$ $\inf \{A(x), A(y)\} \quad(\forall\langle x, y\rangle \in E) ; A$ is called the $m$-polar fuzzy vertex set of $V$ and $B$ is called the $m$-polar fuzzy edge set of $E$. An $m$-polar fuzzy graph $G=(A, B)$ with an underlying pair $(V, E)$ and satisfying $B(\langle x, y\rangle)=B(\langle y, x\rangle)(\forall\langle x, y\rangle \in E)$ and $B(\langle x, x\rangle)=\mathbf{0}(\forall x \in V)$ is called a simple $m$-polar fuzzy graph, where $\mathbf{0}$ is the smallest element of $[0,1]^{m}$. An $m$-polar fuzzy graph $G=(A, B)$ with an underlying pair $(V, E)$ and satisfying $B(\langle x, y\rangle)=\inf \{A(x), A(y)\}(\forall\langle x, y\rangle \in E)$ is called a strong $m$-polar fuzzy graph. The complement of a strong $m$ polar fuzzy graph $G=(A, B)$ (which has an underlying pair $(V, E))$ is a strong $m$-polar fuzzy graph $\bar{G}=(A, \bar{B})$ with an underlying pair $(V, E)$, where $\bar{B}: E \rightarrow[0,1]^{m}$ is defined by $(\langle x, y\rangle \in E, i \in m)$

$$
\begin{aligned}
p_{i} & \circ \bar{B}(\langle x, y\rangle) \\
& = \begin{cases}0, & p_{i} \circ B(\langle x, y\rangle)>0, \\
\inf \left\{p_{i} \circ A(x), p_{i} \circ A(y)\right\}, & p_{i} \circ B(\langle x, y\rangle)=0 .\end{cases}
\end{aligned}
$$

Give two $m$-polar fuzzy graphs (with underlying pairs $\left(V_{1}, E_{1}\right)$ and $\left(V_{2}, E_{2}\right)$, resp. $) G_{1}=\left(A_{1}, B_{1}\right)$ and $G_{2}=\left(A_{2}, B_{2}\right)$. A homomorphism from $G_{1}$ to $G_{2}$ is a mapping $f: V_{1} \rightarrow V_{2}$ which satisfies $A_{1}(x) \leq A_{2}(f(x))\left(\forall x \in V_{1}\right)$ and $B_{1}(\langle x, y\rangle) \leq$ $B_{2}(\langle f(x), f(y)\rangle)\left(\forall\langle x, y\rangle \in E_{1}\right)$. An isomorphism from $G_{1}$ to $G_{2}$ is a bijective mapping $f: V_{1} \rightarrow V_{2}$ which 
satisfies $A_{1}(x)=A_{2}(f(x))\left(\forall x \in V_{1}\right)$ and $B_{1}(\langle x, y\rangle)=$ $B_{2}(\langle f(x), f(y)\rangle)\left(\forall\langle x, y\rangle \in E_{1}\right)$. A weak isomorphism from $G_{1}$ to $G_{2}$ is a bijective mapping $f: V_{1} \rightarrow V_{2}$ which is a homomorphism and satisfies $A_{1}(x)=A_{2}(f(x))\left(\forall x \in V_{1}\right)$. A strong $m$-polar fuzzy graph $G$ is called self-complementary if $G \simeq \bar{G}$ (i.e., there exists an isomorphism between $G$ and its complement $\bar{G})$.

It is not difficult to verify the following conclusions (some of which generalize the corresponding results in $[1,45])$.

(1) In a self-complementary strong $m$-polar fuzzy graph $G=(A, B)$ (with an underlying pair $(V, E))$, we have

$$
\begin{aligned}
& p_{i} \circ \bar{B}(\langle x, y\rangle) \\
& =\inf \left\{p_{i} \circ A(x), p_{i} \circ A(y)\right\}-p_{i} \circ B(\langle x, y\rangle) \\
& \qquad(i \in m,\langle x, y\rangle \in E), \\
& \sum_{x \neq y} p_{i} \circ B(\langle x, y\rangle) \\
& =\frac{1}{2} \sum_{x \neq y} \inf \left\{p_{i} \circ A(x), p_{i} \circ A(y)\right\} \quad(i \in m) .
\end{aligned}
$$

(2) A strong $m$-polar fuzzy graph $G=(A, B)$ (with an underlying pair $(V, E))$ is self-complementary if and only if it satisfies

$$
\begin{array}{r}
p_{i} \circ B(\langle x, y\rangle)=\frac{1}{2} \inf \left\{p_{i} \circ A(x), p_{i} \circ A(y)\right\} \\
(\forall i \in m, \forall\langle x, y\rangle \in E) .
\end{array}
$$

(3) If $G_{1}$ and $G_{2}$ are strong $m$-polar fuzzy graphs, then $G_{1} \simeq G_{2}$ if and only if $\overline{G_{1}} \simeq \overline{G_{2}}$.

(4) Let $G_{1}$ and $G_{2}$ be strong $m$-polar fuzzy graphs. If there is a weak isomorphism from $G_{1}$ to $G_{2}$, then there is a weak isomorphism from $\overline{G_{2}}$ to $\overline{G_{1}}$.

Remark 8. The fuzzifications or bipolar fuzzifications of some algebraic concepts (such as group, $K$-algebra, incline algebra (cf. [48]), ideal, filter, and finite state machine) can be generalized to the case of $m$-polar fuzzy sets. An $m$ polar fuzzy set $A: G \rightarrow[0,1]^{m}$ is called an $m$-polar fuzzy subgroup of a group $(G, \circ)$ if it satisfies $A\left(x \circ y^{-1}\right) \geq$ $\inf \{A(x), A(y)\}(\forall x, y \in G)$. An $m$-polar fuzzy set $A: G \rightarrow$ $[0,1]^{m}$ is called an $m$-polar fuzzy subalgebra of a $K$-algebra $(G, \circ, e, \odot)$ if it satisfies $A(x \circ y) \geq \inf \{A(x), A(y)\}(\forall x, y \in$ $G)$. An $m$-polar fuzzy set $A: X \rightarrow[0,1]^{m}$ is called an $m$-polar fuzzy subincline of an incline $(X,+, *)$ if it satisfies $(x * y) \geq \inf \{A(x), A(y)\}(\forall x, y \in G)$; it is called an $m$ polar fuzzy ideal (resp., an $m$-polar fuzzy filter) of $(X,+, *)$ if it is an $m$-polar fuzzy subincline of $(X,+, *)$ and satisfies $A(x) \geq A(y)$ whenever $x \leq y$ (resp., satisfies $A(x) \leq A(y)$ whenever $x \leq y$ ). An $m$-polar fuzzy finite state machine is a triple $M=(Q, X, A)$, where $Q$ and $X$ are finite nonempty sets (called the set of states and the set of input symbols, resp.) and $A: Q \times X \times Q \rightarrow[0,1]^{m}$ is any $m$-polar fuzzy set on $Q \times X \times Q$. Moreover, if $B: Q \rightarrow[0,1]^{m}$ is an $m$-polar fuzzy set on $Q$ satisfying

$$
\begin{array}{r}
B(q) \geq \inf \{B(p), A(\langle p, x, q\rangle)\} \\
(\forall\langle p, x, q\rangle \in Q \times X \times Q),
\end{array}
$$

then $M_{0}=(Q, X, A, B)$ is called an $m$-polar subsystem of $M$. Furthermore, let $X^{*}$ be the set of all words of elements of $X$ of finite length and $\lambda$ be the empty word in $X^{*}$ (cf. [28]). Then one can define a $m$-polar fuzzy set $A^{*}: Q \times X^{*} \times Q \rightarrow[0,1]^{m}$ on $Q \times X^{*} \times Q$ by putting

$$
\begin{gathered}
A^{*}(\langle q, \lambda, p\rangle)= \begin{cases}\mathbf{1}, & \text { if } q=p, \\
\mathbf{0}, & \text { if } q \neq p,\end{cases} \\
A^{*}(\langle q, \mathbf{x}, x, p\rangle)=\sup _{r \in \mathrm{Q}}\left\{A^{*}(\langle q, x, r\rangle), A^{*}(\langle r, x, p\rangle)\right\} \\
\left(\forall\langle q, \mathbf{x}, x, p\rangle \in Q \times X^{*} \times X \times Q\right),
\end{gathered}
$$

where 1 is the biggest element of $[0,1]^{m}$.

The following conclusions hold.

(1) An $m$-polar fuzzy set $A: G \rightarrow[0,1]^{m}$ is an $m$-polar fuzzy subgroup of a group $(G, \circ)$ if and only if $A_{[a]}=$ $\{x \in G \mid A(x) \geq a\}$ is $\emptyset$ or $A_{[a]}$ is a subgroup of $(G, \circ)\left(\forall a \in[0,1]^{m}\right)$.

(2) An $m$-polar fuzzy set $A: G \rightarrow[0,1]^{m}$ is an $m$-polar fuzzy subalgebra of a $K$-algebra $(G, \circ, e, \odot)$ if and only if $A_{[a]}=\{x \in G \mid A(x) \geq a\}$ is $\emptyset$ or $A_{[a]}$ is a subalgebra of $(G, \circ, e, \odot)\left(\forall a \in[0,1]^{m}\right)$.

(3) An $m$-polar fuzzy set $A: X \rightarrow[0,1]^{m}$ is an $m$ polar fuzzy subincline (resp., an $m$-polar fuzzy ideal, an $m$-polar fuzzy filter) of an incline $(X,+, *)$ if and only if $A_{[a]}$ is a subincline (resp., ideal, filter) of $(X,+, *)\left(\forall a \in[0,1]^{m}\right)$.

(4) Let $M=(Q, X, A)$ be an $m$-polar fuzzy finite state machine and $B: Q \rightarrow[0,1]^{m}$ be an $m$-polar fuzzy set on $Q$. Then $(Q, X, A, B)$ is an $m$-polar subsystem of $M$ if and only if $B(q) \geq$ $\inf \left\{B(p), A^{*}(\langle p, \mathbf{x}, q\rangle)\right\}\left(\forall\langle p, \mathbf{x}, q\rangle \in Q \times X^{*} \times Q\right)$. Please see $[49,50]$ for more results.

Next we consider the applications of $m$-polar fuzzy sets in real world problems.

Example 9. Let $X$ be a set consisting of five patients $x$, $y, z, u$, and $v$ (thus $X=\{x, y, z, u, v\}$ ). They have diagnosis data consisting of three aspects, diagnosis datum of $x$ is $(x)=\langle 0.49,0.46,0.51\rangle$, where datum 0.5 represents "normal" or "OK." Suppose $A(y)=\langle 0.45,0.42,0.59\rangle$, $A(z)=\langle 0.50,0.40,0.54\rangle, A(u)=\langle 0.40,0.49,0.60\rangle$, and $A(v)=\langle 0.51,0.52,0.50\rangle$. Then we obtain a 3 -polar fuzzy 
set $A: X \rightarrow[0,1]^{3}$ which can describe the situation; this 3 -polar fuzzy set can also be written as follows:

$$
\begin{aligned}
A=\left\{\frac{\langle 0.49,0.46,0.51\rangle}{x}, \frac{\langle 0.45,0.42,0.59\rangle}{y}, \frac{\langle 0.50,0.40,0.54\rangle}{z},\right. \\
\left.\frac{\langle 0.40,0.49,0.60\rangle}{u}, \frac{\langle 0.51,0.52,0.50\rangle}{v}\right\} .
\end{aligned}
$$

Example 10. $m$-polar fuzzy sets can be used in decision making. In many decision making situations, it is necessary to gather the group consensus. This happens when a group of friends decides which movie to watch, when a company decides which product design to manufacture, and when a democratic country elects its leaders. For instance, we consider here only the case of election. Let $X=\{x, y, z, \ldots, u, v\}$ be the set of voters and $C=\left\{c_{1}, c_{2}, c_{3}, c_{4}\right\}$ be the set of all the four candidates. Suppose the voting is weighted. For each candidate $c \in C$, a voter in $\{x, y, z\}$ can send a value in $[0,1]$ to $c$, but a voter in $X-\{x, y, z\}$ can only send a value in $[0.1,0.8]$ to $c$. Suppose $A(x)=\langle 0.9,0.4,0.01,0.1\rangle$ (which means the preference degrees of $x$ corresponding to $c_{1}, c_{2}, c_{3}$, and $c_{4}$ are $0.9,0.4,0.01$, and 0.1 , resp.), $A(y)=\langle 0.2,0.3,0.8,0.1\rangle$, $A(z)=\langle 0.8,0.9,0.8,0.2\rangle, \ldots, A(u)=\langle 0.6,0.8,0.8,0.1\rangle$, and $A(v)=\langle 0.7,0.8,0.4,0.2\rangle$. Then we obtain a 4 -polar fuzzy set $A: X \rightarrow[0,1]^{4}$ which can describe the situation; this 4polar fuzzy set can also be written as follows:

$$
\begin{aligned}
& A=\left\{\frac{\langle 0.9,0.4,0.01,0.1\rangle}{x}, \frac{\langle 0.2,0.3,0.8,0.1\rangle}{y},\right. \\
& \frac{\langle 0.8,0.9,0.8,0.2\rangle}{z}, \ldots, \frac{\langle 0.6,0.8,0.8,0.1\rangle}{u}, \\
&\left.\frac{\langle 0.7,0.8,0.4,0.2\rangle}{v}\right\} .
\end{aligned}
$$

Example 11. $m$-polar fuzzy sets can be used in cooperative games (cf. [51]). Let $X=\left\{x_{1}, x_{2}, \ldots, x_{n}\right\}$ be the set of $n$ agents or players $(n \geq 1), m=\{0,1, \ldots, m-1\}$ be the set of the grand coalitions, and $A: X \rightarrow[0,1]^{m}$ be an $m$-polar fuzzy set, where $p_{i} \circ A(x)$ is the degree of player $x$ participating in coalition $i(x \in X, i \in m)$. Again let $v:[0,1]^{m} \rightarrow R$ (the set of all real numbers) be a mapping satisfying $v(\mathbf{0})=0$. Then the mapping $v \circ A: X \rightarrow R$ is called a cooperative game, where $v \circ A(x)$ represents the amount of money obtained by player $x$ under the coalition participating ability $A(x)(x \in X)$.

(1) (a public good game; compare with [51, Example 6.5]) Suppose $n$ agents $x_{1}, x_{2}, \ldots, x_{n}$ want to create a facility for joint use. The cost of the facility depends on the sum of the participation levels (or degrees) of the agents and it is described by

$$
k\left(\sum_{i=1}^{n} B\left(x_{i}\right)\right)
$$

where $k:[0, n] \rightarrow R$ is a continuous monotonic increasing function with $k(0)=0$ and $B: X \rightarrow[0,1]$ is a mapping. Let
$A: X=\left\{x_{1}, x_{2}, \ldots, x_{n}\right\} \rightarrow[0,1]^{n}$ be a mapping satisfying $p_{j} \circ A\left(x_{i}\right)=B\left(x_{i}\right)$ (if $j=i$ ) or 0 (otherwise) $(i=1,2, \ldots, n)$. Then a cooperative game model $v \circ A: X \rightarrow R$ is established, where $v:[0,1]^{n} \rightarrow R$ is defined by

$$
\begin{aligned}
v\left(\left\langle s_{1}, s_{2}, \ldots, s_{n}\right\rangle\right)= & \sum_{i=1}^{n} g_{i}\left(s_{i}\right)-\frac{1}{n} k\left(\sum_{i=1}^{n} B\left(x_{i}\right)\right) \\
& \left(\forall\left\langle s_{1}, s_{2}, \ldots, s_{n}\right\rangle \in[0,1]^{n}\right),
\end{aligned}
$$

and the function $g_{i}:[0,1] \rightarrow R$ is continuously monotonic increasing with $g_{i}(0)=0(i=1,2, \ldots, n)$. Obviously, the gain of agent $x_{i}$ (with participation level $B\left(x_{i}\right)$ ) is

$$
v \circ A\left(x_{i}\right)=g_{i} \circ B\left(x_{i}\right)-\frac{1}{n} k\left(\sum_{i=1}^{n} B\left(x_{i}\right)\right),
$$

and the total gain is

$$
\sum_{i=1}^{n} v \circ A\left(x_{i}\right)=\sum_{i=1}^{n} g_{i} \circ B\left(x_{i}\right)-k\left(\sum_{i=1}^{n} B\left(x_{i}\right)\right) .
$$

(2) There are two goods, denoted $g_{1}$ and $g_{2}$, and three agents $a, b$, and $c$ with endowments $(\varepsilon, \varepsilon),(1-\varepsilon, 0)$, and $(0,1-\varepsilon)(0<\varepsilon \leq 1)$. Let $v:[0,1]^{2} \rightarrow R$ be any mapping satisfying $v(\langle 0,0\rangle)=0$. Then the corresponding cooperative game model is $v \circ A: X=\{a, b, c\} \rightarrow R$, where

$$
\begin{gathered}
A=\left\{\frac{\langle\varepsilon, \varepsilon\rangle}{a}, \frac{\langle 1-\varepsilon, 0\rangle}{b}, \frac{\langle 0,1-\varepsilon\rangle}{c}\right\}, \\
v \circ A=\left\{\frac{v(\langle\varepsilon, \varepsilon\rangle)}{a}, \frac{v(\langle 1-\varepsilon, 0\rangle)}{b}, \frac{v(\langle 0,1-\varepsilon\rangle)}{c}\right\} .
\end{gathered}
$$

Example 12. $m$-polar fuzzy sets can be used to define weighted games. A weighted game is a 4 -tuple $(X, \mathscr{P}, W, \Delta)$, where $X=\left\{x_{1}, x_{2}, \ldots, x_{n}\right\}$ is the set of $n$ players or voters $(n \geq 2), \mathscr{P}$ is a collection of fuzzy sets on $X$ (called coalitions) such that $(\mathscr{P}, \leq)$ is upper set (i.e., a fuzzy set $Q$ on $X$ belongs to $\mathscr{P}$ if $Q \geq P$ for some $P \in \mathscr{P}), W: X \rightarrow[0,1]^{m}$ is an $m$-polar fuzzy set on $X$ (called voting weights), and $\Delta \subseteq[0,+\infty)^{m}-\{\mathbf{0}\}$ (called quotas). Imagine a situation: three people, $x, y$, and $z$, vote for a proposal on releasing of a student. Suppose that $x$ casts 200 US Dollars and lose 80 hairs on her head votes each, $y$ casts 60000 US Dollars and 100 grams Cordyceps sinensis votes each, $z$ casts 100000 US Dollars and 100 grams gold votes each. Then an associated weighted game model is $(X, \mathscr{P}, W, \Delta)$, where $X=\{x, y, z\}$ and $\mathscr{P}$ is a collection of fuzzy sets on $X$ with $(\mathscr{P}, \leq)$ an upper set, $m=4$,

$$
\begin{aligned}
W= & \left\{\frac{\langle 200 / 160200,80 / 80,0,0\rangle}{x},\right. \\
& \frac{\langle 60000 / 160200,0,100 / 100,0\rangle}{y}, \\
& \left.\frac{\langle 100000 / 160200,0,0,100 / 100\rangle}{z}\right\}, \\
\Delta=\{\langle 200000,0,0,0\rangle,\langle 100000,300,0,0\rangle, & \\
& \langle 0,0,500,0\rangle,\langle 0,0,0,65000\rangle\} .
\end{aligned}
$$


(1) If the situation is a little simple, $x$ casts $[100,300]$ US Dollars (i.e., the cast is between 100 US Dollars and 300 US Dollars, where $[100,300]$ is an interval number which can be looked as a point $[0,+\infty)^{2}$ ) votes each, $y$ casts $[50000,70000]$ US Dollars votes each, $z$ casts $[90000,110000]$ US Dollars votes each, and quota is $[100000,120000]$. Then the corresponding weighted game model is $(X, \mathscr{P}, W,[100000,120000])$, where $\mathscr{P}$ is a collection of fuzzy sets on $X$ with $(\mathscr{P}, \leq)$ an upper set, $m=1$, and

$$
W=\left\{\frac{400 / 320400}{x}, \frac{120000 / 320400}{y}, \frac{200000 / 320400}{z}\right\} .
$$

(2) If the situation is more simple, $x$ casts 200 US Dollars votes each, $y$ casts 60000 US Dollars votes each, $z$ casts 100000 US Dollars votes each, and quota is 110000 . Then the corresponding weighted game model is $(X, \mathscr{P}, W, 110000)$, where $\mathscr{P}=\{\{x, z\},\{y, z\},\{x, y, z\}\}, m=1$, and

$$
W=\left\{\frac{200 / 160200}{x}, \frac{60000 / 160200}{y}, \frac{100000 / 160200}{z}\right\} .
$$

Notice that the subset $\{x, z\} \subseteq X$ is exactly a fuzzy set $A$ : $X \rightarrow[0,1]$ on $X$ defined by $A(x)=A(z)=1$ and $A(y)=0$.

Example 13. $m$-polar fuzzy sets can be used as a model for clustering or classification. Consider a set $X$ consisting of $n$ students $x_{1}, x_{2}, \ldots, x_{n}(n \geq 2)$ in Chinese middle school. For a student $x \in X$, we use integers $x_{1}$ (resp., $x_{2}, \ldots, x_{6}$ ) in $[0,100]$ to denote the average score of Mathematics (resp., Physics, Chemistry, Biology, Chinese, and English), and

$$
\begin{array}{r}
A(x)=\left\langle x_{1} \times 0.01, x_{2} \times 0.01, x_{3} \times 0.01,\right. \\
\left.x_{4} \times 0.01, x_{5} \times 0.01, x_{6} \times 0.01\right\rangle .
\end{array}
$$

Then we obtain a 6-polar fuzzy set model $A: X \rightarrow[0,1]^{6}$, which can be used for clustering or classification of these students.

Example 14. $m$-polar fuzzy sets can be used to define multivalued relations.

(1) Consider a set $X$ consisting of $n$ net users (resp., patients) $x_{1}, x_{2}, \ldots, x_{n}(n \geq 2)$. For net users (resp., patients) $x, y \in X$, we use $(x, y, j)$ to denote the similarity between $x$ and $y$ in $j$ th aspect $(1 \leq$ $j \leq m, m \geq 2)$, and let $A(x, y)=A(y, x)=$ $\langle(x, y, 1),(x, y, 2), \ldots,(x, y, m)\rangle$. Then we obtain an $m$-polar fuzzy set $A: X \rightarrow[0,1]^{m}$, which is a multivalued similarity relation.

(2) Consider a set $X$ consisting of $n$ people $x_{1}, x_{2}$, $\ldots, x_{n}(n \geq 2)$ in a social network. For $x, y \in$ $X$, we use $(x, y, j)$ to denote the degree of connection between $x$ and $y$ in $j$ th aspect $(1 \leq j \leq$ $m, m \geq 2)$, and let $A(x, y)=A(y, x)=\langle(x, y, 1)$, $(x, y, 2), \ldots,(x, y, m)\rangle$. Then we obtain an $m$-polar fuzzy set $A: X \rightarrow[0,1]^{m}$, which is a multivalued social graph (or multivalued social network) model.

\section{Conclusion}

In this note, we show that the enthusiastically studied notion of bipolar fuzzy set is actually a synonym of a $[0,1]^{2}$-set (we call it 2-polar fuzzy set), and thus we highlight the notion of $m$-polar fuzzy set (actually a $[0,1]^{m}$-set, $m \geq 2$ ). The $m$-polar fuzzy sets not only have real backgrounds (e.g., "multipolar information" exists) but also have applications in both theory and real world problems (which have been illustrated by examples).

\section{Conflict of Interests}

The authors declare that there is no conflict of interests regarding the publication of this paper.

\section{Acknowledgments}

This work was supported by the International Science and Technology Cooperation Foundation of China (Grant no. 2012DFA11270) and the National Natural Science Foundation of China (Grant no. 11071151).

\section{References}

[1] L. A. Zadeh, "Fuzzy sets," Information and Control, vol. 8, no. 3, pp. 338-353, 1965.

[2] U. Höhle and S. E. Rodabaugh, Eds., Mathematics of Fuzzy Sets: Logic, Topology, and Measure Theory, The Handbooks of Fuzzy Sets Series, Kluwer Academic, Dordrecht, The Netherlands, 1999.

[3] W. R. Zhang, "Bipolar fuzzy sets and relations: a computational framework for cognitive modeling and multiagent decision analysis," in Proceedings of the Industrial Fuzzy Control and Intelligent Systems Conference, and the NASA Joint Technology Workshop on Neural Networks and Fuzzy Logic and Fuzzy Information Processing Society Biannual Conference, pp. 305309, San Antonio, Tex, USA, December 1994.

[4] M. Akram, "Bipolar fuzzy graphs," Information Sciences, vol. 181, no. 24, pp. 5548-5564, 2011.

[5] M. Akram, "Bipolar fuzzy graphs with applications," KnowledgeBased Systems, vol. 39, pp. 1-8, 2013.

[6] M. Akram, W. Chen, and Y. Yin, "Bipolar fuzzy Lie superalgebras," Quasigroups and Related Systems, vol. 20, no. 2, pp. 139156, 2012.

[7] M. Akram, S. G. Li, and K. P. Shum, "Antipodal bipolar fuzzy graphs," Italian Journal of Pure and Applied Mathematics, vol. 31, pp. 425-438, 2013.

[8] M. Akram, A. B. Saeid, K. P. Shum, and B. L. Meng, "Bipolar fuzzy K-algebras," International Journal of Fuzzy Systems, vol. 12, no. 3, pp. 252-259, 2010.

[9] L. Amgoud, C. Cayrol, M. C. Lagasquie-Schiex, and P. Livet, "On bipolarity in argumentation frameworks," International Journal of Intelligent Systems, vol. 23, no. 10, pp. 1062-1093, 2008.

[10] H. Y. Ban, M. J. Kim, and Y. J. Park, "Bipolar fuzzy ideals with operators in semigroups," Annals of Fuzzy Mathematics and Informatics, vol. 4, no. 2, pp. 253-265, 2012.

[11] S. Benferhat, D. Dubois, S. Kaci, and H. Prade, "Bipolar possibility theory in preference modeling: representation, fusion and 
optimal solutions," Information Fusion, vol. 7, no. 1, pp. 135-150, 2006.

[12] S. Bhattacharya and S. Roy, "Study on bipolar fuzzy-rough control theory," International Mathematical Forum, vol. 7, no. 41, pp. 2019-2025, 2012.

[13] I. Bloch, "Dilation and erosion of spatial bipolar fuzzy sets," in Applications of Fuzzy Sets Theory, F. Masulli, S. Mitra, and G. Pasi, Eds., vol. 4578 of Lecture Notes in Computer Science, pp. 385-393, Springer, Berlin, Germany, 2007.

[14] I. Bloch, "Bipolar fuzzy spatial information: geometry, morphology, spatial reasoning," in Methods for Handling Imperfect Spatial Information, R. Jeansoulin, O. Papini, H. Prade, and S. Schockaert, Eds., vol. 256 of Studies in Fuzziness and Soft Computing, pp. 75-102, Springer, Berlin, Germany, 2010.

[15] I. Bloch, "Lattices of fuzzy sets and bipolar fuzzy sets, and mathematical morphology," Information Sciences, vol. 181, no. 10, pp. 2002-2015, 2011.

[16] I. Bloch, "Mathematical morphology on bipolar fuzzy sets: general algebraic framework," International Journal of Approximate Reasoning, vol. 53, no. 7, pp. 1031-1060, 2012.

[17] I. Bloch and J. Atif, "Distance to bipolar information from morphological dilation," in Proceedings of the 8th Conference of the European Society for Fuzzy Logic and Technology, pp. 266273, 2013.

[18] J. F. Bonnefon, "Two routes for bipolar information processing, and a blind spot in between," International Journal of Intelligent Systems, vol. 23, no. 9, pp. 923-929, 2008.

[19] P. Bosc and O. Pivert, "On a fuzzy bipolar relational algebra," Information Sciences, vol. 219, pp. 1-16, 2013.

[20] D. Dubois, S. Kaci, and H. Prade, "Bipolarity in reasoning and decision, an introduction," in Proceedings of the International Conference on Information Processing and Management of Uncertainty, pp. 959-966, 2004.

[21] D. Dubois and H. Prade, "An overview of the asymmetric bipolar representation of positive and negative information in possibility theory," Fuzzy Sets and Systems, vol. 160, no. 10, pp. 1355-1366, 2009.

[22] U. Dudziak and B. Pękala, "Equivalent bipolar fuzzy relations," Fuzzy Sets and Systems, vol. 161, no. 2, pp. 234-253, 2010.

[23] H. Fargier and N. Wilson, "Algebraic structures for bipolar constraint-based reasoning," in Symbolic and Quantitative Approaches to Reasoning with Uncertainty, vol. 4724 of Lecture Notes in Computer Science, pp. 623-634, Springer, Berlin, Germany, 2007.

[24] M. Grabisch, S. Greco, and M. Pirlot, "Bipolar and bivariate models in multicriteria decision analysis: descriptive and constructive approaches," International Journal of Intelligent Systems, vol. 23, no. 9, pp. 930-969, 2008.

[25] M. M. Hasankhani and A. B. Saeid, "Hyper MV-algebras defined by bipolar-valued fuzzy sets," Annals of West University of Timisoara-Mathematics, vol. 50, no. 1, pp. 39-50, 2012.

[26] C. Hudelot, J. Atif, and I. Bloch, "Integrating bipolar fuzzy mathematical morphology in description logics for spatial reasoning," Frontiers in Artificial Intelligence and Applications, vol. 215, pp. 497-502, 2010.

[27] Y. B. Jun, M. S. Kang, and H. S. Kim, "Bipolar fuzzy hyper BCKideals in hyper BCK-algebras," Iranian Journal of Fuzzy Systems, vol. 8, no. 2, pp. 105-120, 2011.

[28] Y. B. Jun and J. Kavikumar, "Bipolar fuzzy finite state machines," Bulletin of the Malaysian Mathematical Sciences Society, vol. 34, no. 1, pp. 181-188, 2011.
[29] Y. B. Jun, H. S. Kim, and K. J. Lee, "Bipolar fuzzy translation in BCK/BCI-algebra," Journal of the Chungcheong Mathematical Society, vol. 22, no. 3, pp. 399-408, 2009.

[30] Y. B. Jun and C. H. Park, "Filters of BCH-algebras based on bipolar-valued fuzzy sets," International Mathematical Forum, vol. 4, no. 13, pp. 631-643, 2009.

[31] S. Kaci, "Logical formalisms for representing bipolar preferences," International Journal of Intelligent Systems, vol. 23, no. 9, pp. 985-997, 2008.

[32] K. J. Lee, "Bipolar fuzzy subalgebras and bipolar fuzzy ideals of BCK/BCI-algebras," Bulletin of the Malaysian Mathematical Sciences Society, vol. 32, no. 3, pp. 361-373, 2009.

[33] K. J. Lee and Y. B. Jun, "Bipolar fuzzy a-ideals of BCI-algebras," Communications of the Korean Mathematical Society, vol. 26, no. 4, pp. 531-542, 2011.

[34] K. M. Lee, "Comparison of interval-valued fuzzy sets, intuitionistic fuzzy sets, and bipolar-valued fuzzy sets," Journal of Fuzzy Logic Intelligent Systems, vol. 14, no. 2, pp. 125-129, 2004.

[35] R. Muthuraj and M. Sridharan, "Bipolar anti fuzzy HX group and its lower level sub HX groups," Journal of Physical Sciences, vol. 16, pp. 157-169, 2012.

[36] S. Narayanamoorthy and A. Tamilselvi, "Bipolar fuzzy line graph of a bipolar fuzzy hypergraph," Cybernetics and Information Technologies, vol. 13, no. 1, pp. 13-17, 2013.

[37] E. Raufaste and S. Vautier, "An evolutionist approach to information bipolarity: representations and affects in human cognition," International Journal of Intelligent Systems, vol. 23, no. 8, pp. 878-897, 2008.

[38] A. B. Saeid, "BM-algebras defined by bipolar-valued sets," Indian Journal of Science and Technology, vol. 5, no. 2, pp. 20712078, 2012.

[39] S. Samanta and M. Pal, "Irregular bipolar fuzzy graphs," International Journal of Applications of Fuzzy Sets, vol. 2, no. 2, pp. 91-102, 2012.

[40] H. L. Yang, S. G. Li, Z. L. Guo, and C. H. Ma, "Transformation of bipolar fuzzy rough set models," Knowledge-Based Systems, vol. 27, pp. 60-68, 2012.

[41] H. L. Yang, S. G. Li, S. Y. Wang, and J. Wang, "Bipolar fuzzy rough set model on two different universes and its application," Knowledge-Based Systems, vol. 35, pp. 94-101, 2012.

[42] W. R. Zhang, "Equilibrium relations and bipolar fuzzy clustering," in Proceedings of the 18th International Conference of the North American Fuzzy Information Processing Society (NAFIPS '99), pp. 361-365, June 1999.

[43] W. R. Zhang, Ed., Yin Yang Bipolar Relativity: A Unifying Theory of Nature, Agents and Causality with Applications in Quantum Computing, Cognitive Informatics and Life Sciences, IGI Global, 2011.

[44] W. R. Zhang, "Bipolar quantum logic gates and quantum cellular combinatorics-a logical extension to quantum entanglement," Journal of Quantum Information Science, vol. 3, no. 2, pp. 93-105, 2013.

[45] H. L. Yang, S. G. Li, W. H. Yang, and Y. Lu, "Notes on 'bipolar fuzzy graphs," Information Sciences, vol. 242, pp. 113-121, 2013.

[46] A. Rosenfeld, "Fuzzy graphs," in Fuzzy Sets and Their Applications to Cognitive and Decision Process, L. A. Zadeh, K. S. Fu, and M. Shimura, Eds., pp. 77-95, Academic Press, New York, NY, USA, 1975.

[47] R. T. Yeh and S. Y. Bang, "Fuzzy relations, fuzzy graphs and their application to clustering analysis," in Fuzzy Sets and Their Applications to Cognitive and Decision Process, L. A. Zadeh, K. S. Fu, 
and M. Shimura, Eds., pp. 338-353, Academic Press, New York, NY, USA, 1975.

[48] Z. Q. Cao, K. H. Kim, and F. W. Roush, Incline Algebra and Applications, Ellis Horwood Series in Mathematics and Its Applications, Halsted Press, Chichester, UK; John Wiley \& Sons, New York, NY, USA, 1984.

[49] Y. M. Li, "Finite automata theory with membership values in lattices," Information Sciences, vol. 181, no. 5, pp. 1003-1017, 2011.

[50] J. H. Jin, Q. G. Li, and Y. M. Li, "Algebraic properties of $L$ fuzzy finite automata," Information Sciences, vol. 234, pp. 182202, 2013.

[51] L. J. Xie and M. Grabisch, "The core of bicapacities and bipolar games," Fuzzy Sets and Systems, vol. 158, no. 9, pp. 1000-1012, 2007. 


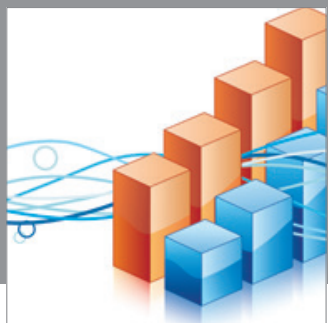

Advances in

Operations Research

mansans

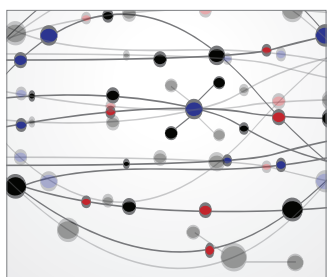

The Scientific World Journal
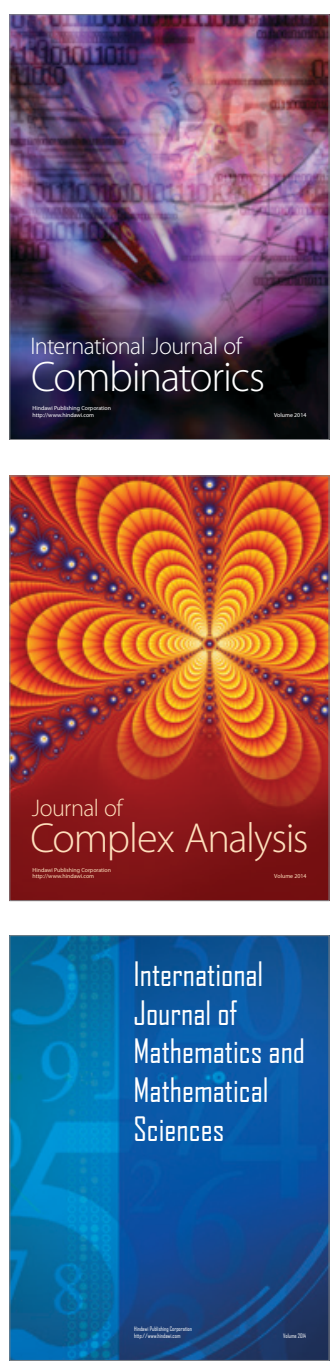
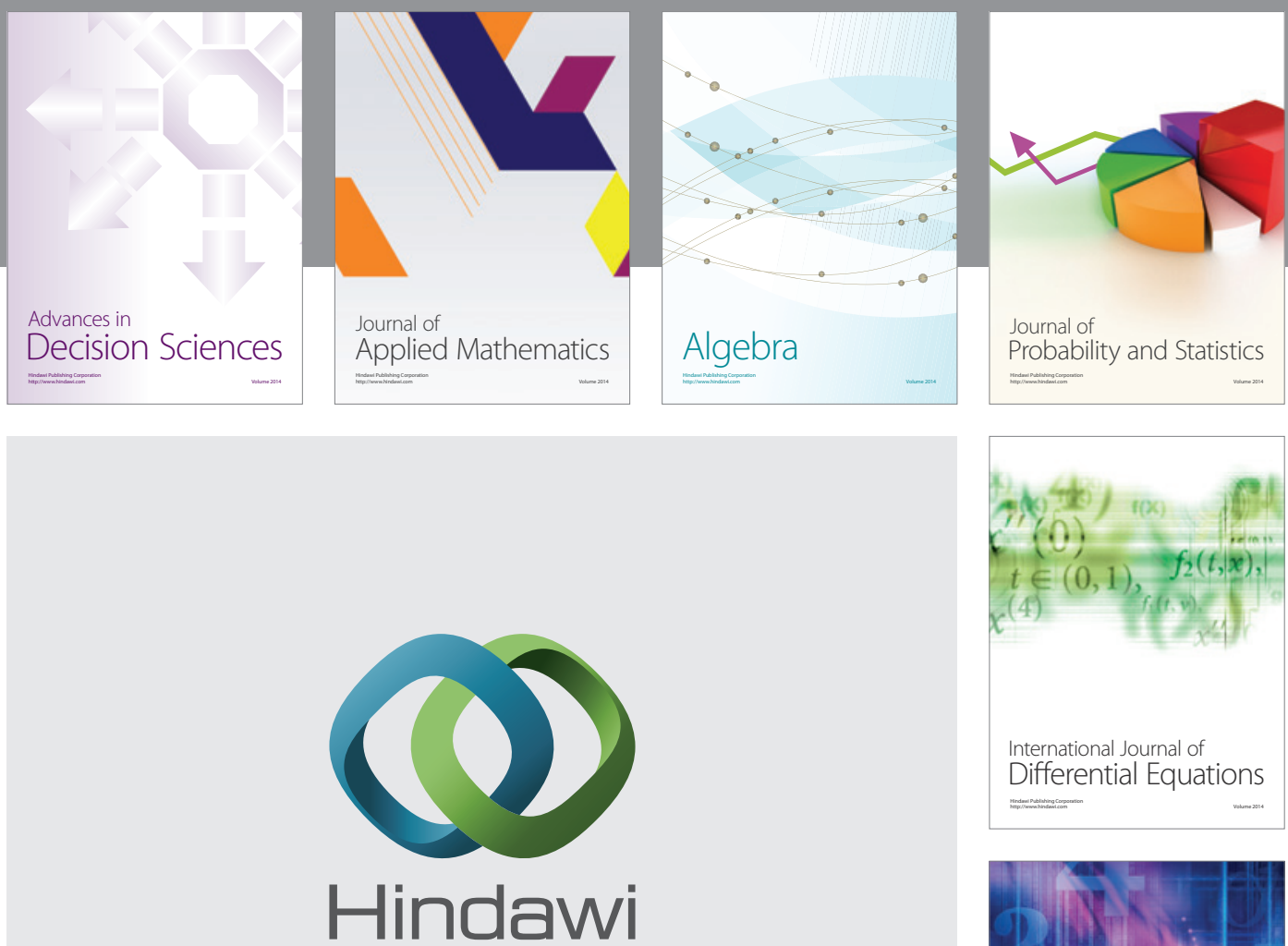

Submit your manuscripts at http://www.hindawi.com
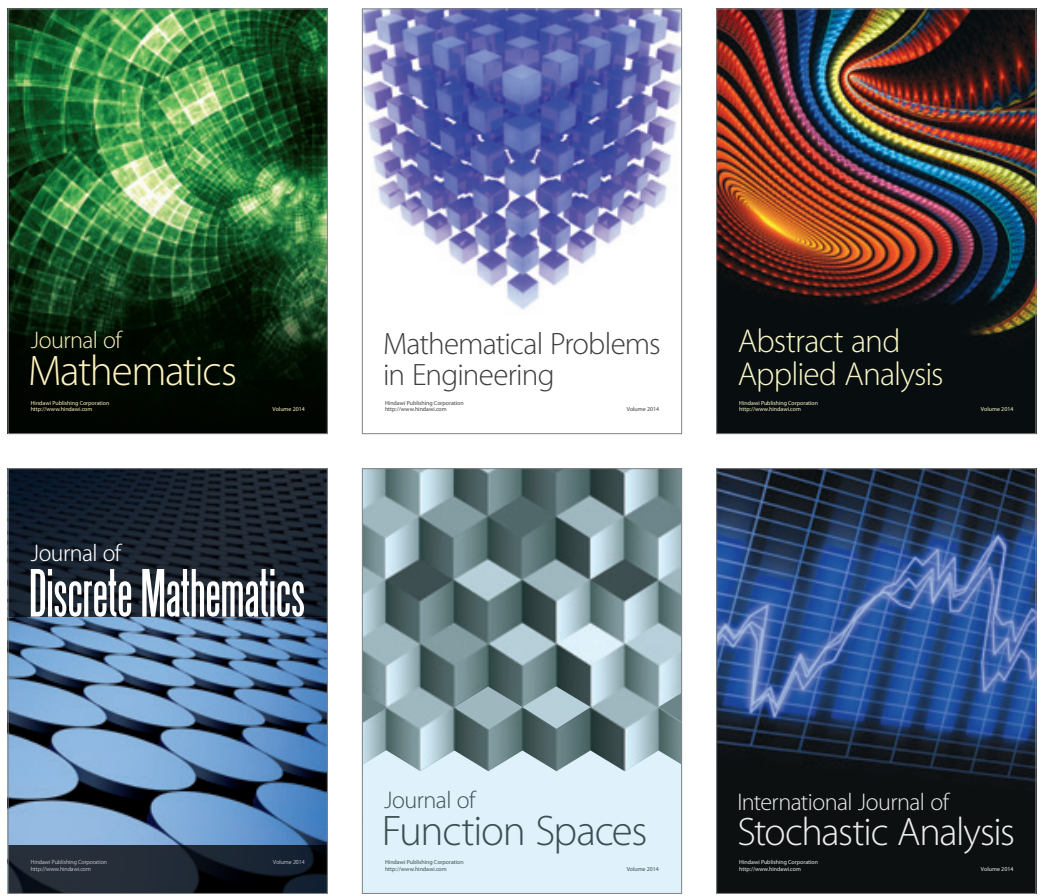

Journal of

Function Spaces

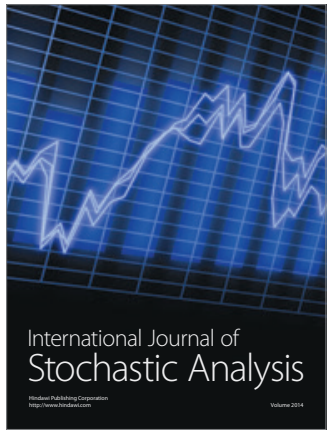

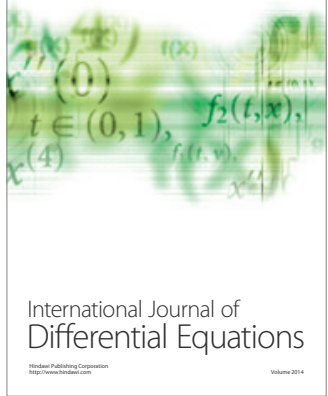
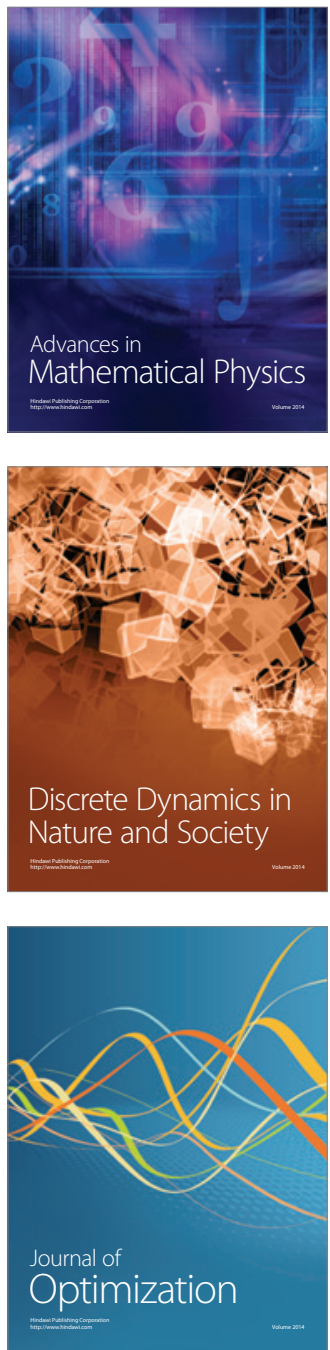